\title{
Determining the Influence of Granule Size on Simulation Parameters and Residual Shear Stress Distribution in Tablets by Combining the Finite Element Method into the Design of Experiments
}

\author{
Yoshihiro Hayashi, ${ }^{*, a}$ Atsushi Kosugi, ${ }^{b}$ Takahiro Miura, ${ }^{c}$ Kozo Takayama, ${ }^{d}$ and Yoshinori Onuki ${ }^{a}$ \\ ${ }^{a}$ Department of Pharmaceutical Technology, Graduate School of Medicine and Pharmaceutical Science for \\ Research, University of Toyama; 2630 Sugitani, Toyama 930-0194, Japan: ${ }^{b}$ Formulation Development Department, \\ Development \& Planning Division, Nichi-Iko Pharmaceutical Co., Ltd.; 205-1 Shimoumezawa, Namerikawa, Toyama \\ 936-0857, Japan: ${ }^{c}$ Mechanical CAE Division, Cybernet Systems Co., Ltd.; FUJISOFT Bldg. 3 Kanda-neribeicho, \\ Chiyoda-ku, Tokyo 101-0022, Japan: and ${ }^{d}$ Faculty of Pharmacy and Pharmaceutical Sciences, Josai University; 1-1 \\ Keyakidai, Sakado, Saitama 350-0295, Japan. \\ Received November 28, 2017; accepted February 1, 2018
}

The influence of granule size on simulation parameters and residual shear stress in tablets was determined by combining the finite element method (FEM) into the design of experiments (DoE). Lactose granules were prepared using a wet granulation method with a high-shear mixer and sorted into small and large granules using sieves. To simulate the tableting process using the FEM, parameters simulating each granule were optimized using a DoE and a response surface method (RSM). The compaction behavior of each granule simulated by FEM was in reasonable agreement with the experimental findings. Higher coefficients of friction between powder and die/punch $(\mu)$ and lower by internal friction angle $\left(\alpha^{y}\right)$ were generated in the case of small granules, respectively. RSM revealed that die wall force was affected by $\alpha^{y}$. On the other hand, the pressure transmissibility rate of punches value was affected not only by the $\alpha^{y}$ value, but also by $\mu$. The FEM revealed that the residual shear stress was greater for small granules than for large granules. These results suggest that the inner structure of a tablet comprising small granules was less homogeneous than that comprising large granules. To evaluate the contribution of the simulation parameters to residual stress, these parameters were assigned to the fractional factorial design and an ANOVA was applied. The result indicated that $\mu$ was the critical factor influencing residual shear stress. This study demonstrates the importance of combining simulation and statistical analysis to gain a deeper understanding of the tableting process.

Key words tablet; finite element method; granulation; particle size; residual stress distribution

Designing and testing pharmaceutical products in silico have gained attention in pharmaceutical development. This approach might reduce the expense of experimental and validation experiments. ${ }^{1)}$ The finite element method (FEM) is a numerical method that is increasingly used for simulating the compaction behavior of pharmaceutical powders. ${ }^{2-4)}$ The FEM models powders as continuum media and the compaction behavior is analyzed by solving boundary value problems. Before the FEM analysis, it is necessary to define the mechanical behavior of pharmaceutical powders during compaction. The Drucker-Prager cap (DPC) model has been widely used for modeling the mechanical behavior of powders because the method can represent the densification, hardening, and interparticle frictions of the powders. ${ }^{5,6)}$

The FEM, in which the powder is modeled using the DPC model, is the most widely used for modeling the compaction behavior of metals, cosmetics, and pharmaceutical powders. ${ }^{5,7,8)}$ For instance, the relative density distribution of tablets can be estimated using the FEM and DPC model. ${ }^{2}$ FEM results were consistent with the results obtained using an indentation hardness tester. Residual stress distribution of tablets composed of lactose can be calculated using the FEM. ${ }^{4}$ The capping tendency might be caused by the intense shear band and the coefficient of friction has an influence on the intensity of shear stress. A three-dimensional FEM was used to simulate the variation of density distribution during compaction for double-radius and single-radius tablets. ${ }^{5)}$ The variation in plastic strain of tablets during diametrical compression was determined. The variation in plastic strain inside of tablets is a source of cracking and can be a cause of the capping tendency during the diametrical compression tests.

FEM analysis is a powerful tool for understanding compaction behavior. However, it is difficult to clarify the relationships between simulation parameters and obtained results because there are many variables to consider, including material characteristics, e.g., DPC model parameters, and boundary conditions, e.g., coefficients of friction and initial state. Statistical techniques have been employed to overcome this difficulty. There is an approach used in various simulation conditions and to evaluate simulation results obtained using statistical techniques. ${ }^{9)}$ This approach enables clarification of the relationships between simulation parameters and results. Therefore, we can infer the critical factors that influence the quality of products based on data obtained in silico. For these reasons, a combination of simulation and statistical methods might facilitate new insights into pharmaceutical product design.

Previously, we suggested that simulation parameters and residual stress distribution of tablets are influenced by process parameters of high-shear wet granulation, such as the amount of water and kneading time. ${ }^{10)}$ Generally, process parameters affect granule characteristics, such as granule size distribution, pore distribution, and granule hardness. These characteristics have an impact on tablet properties such as crushing 
force, friability, and dissolution property. ${ }^{11)}$ Therefore, simulation parameters and residual stress distribution might be influenced by granule characteristics.

The purpose of the present study was to clarify the relationships between granule characteristics, simulation parameters, and residual stress distribution using the FEM and statistical methods. In particular, we have focused on granule size because it strongly affects tablet characteristics such as tensile strength, friability and dissolution property. ${ }^{11-13)}$ To achieve this aim, we prepared model granules using a high-shear granulation method and sorted them into groups of small (less than $355 \mu \mathrm{m}$ ) and large granules (ranging from 710 to $1200 \mu \mathrm{m})$ using sieves. Subsequently, we estimated the simulation and DPC model parameters, which were consistent with experimental results achieved using response surface method and the optimization method. Then, the optimized parameters were entered into ANSYS software, in which the DPC model was implemented. Finally, we estimated residual stress distribution using the FEM in various conditions based on the design of experiments (DoE) and analyzed the data using statistical methods, to clarify the relationships between the granule size, simulation parameters, and residual stress distributions.

\section{Experimental}

Materials Lactose (LAC; Pharamatose 200M, DMV, Veghel, the Netherlands) was purchased. Magnesium stearate (Mg-St) was purchased from Wako Pure Chemical Industries, Ltd. (Osaka, Japan).

Preparation of Granules To exclude the concerns about segregation of specific components in the granules and keep the quality uniform, the test granules were prepared from only lactose by kneading with purified water added as a binder solution.

One kilogram of lactose was granulated using a highspeed mixer (FS-GS-5, Fukae Pawteck Co., Osaka, Japan) for $9 \mathrm{~min}$. Purified water was used as binder and the volume was set at $175 \mathrm{~mL}$. Blade and chopper speeds were set at 500 and $1300 \mathrm{rpm}$, respectively. After the granulation process, the granules were dried at $75^{\circ} \mathrm{C}$ for $50 \mathrm{~min}$ using a box-type dryer (607C, Iwakuro Mfg., Toyama, Japan). The granules were sieved and divided into two fractions, namely, small granules (less than $355 \mu \mathrm{m}$ ) and large ones (ranging from 710 to $1200 \mu \mathrm{m})$. One percent of $\mathrm{Mg}-\mathrm{St}$ was added to each granule fraction and the mixture was blended in a polyethylene bag.

Tableting and Characterizing the Granule Compaction Behavior Granules were compacted using a single tableting machine equipped with an instrumented die (TK-TB20KN-S, Tokushu Keisoku Co., Yokohama, Japan). During the tableting process, the axial forces on the upper and lower punches were recorded with load cells of $20 \mathrm{kN}$ capacity. The displacement of the upper punch and the radial stress were also measured during compaction. Force and displacement transducers have an accuracy of $\pm 0.01 \mathrm{kN}$ and $\pm 10 \mu \mathrm{m}$, respectively. To obtain accurate measurements of the compaction behavior, compression and ejection speeds were set relatively low (compression speed, $0.2 \mathrm{~mm} / \mathrm{s}$; decompression speed, $0.2 \mathrm{~mm} / \mathrm{s})$. We obtained approximately 1870 data points per tableting. The smoothing procedure was performed to reduce random noise. Flat-faced $8 \mathrm{~mm}$ diameter punches were used to prepare tablets containing $200 \mathrm{mg}$ of granules. Granules were manually filled into the die and compressed. The punch force was set at $6 \mathrm{kN}$ without precompression. The measurements of compaction behavior were carried out in triplicate.

Simulation of the Compaction Behavior of Granules Using FEM Because the compaction of cylindrical tablets was performed in an axisymmetric case, it can be analyzed using a two-dimensional FEM. Figure 1 shows the scheme of the FEM. The die wall and upper punch were modeled as rigid bodies. The interaction between the granule, die wall, and upper punch was modeled and, the coefficient of friction $(\mu)$ between the granules and the die was set as a simulation parameter. The initial height of the granule bed $\left(Z_{0}\right)$ was also set as a simulation parameter. The nodes on the symmetry axis were restricted to move only vertically, and the nodes at the bottom boundaries were restricted to move only horizontally. The upper punch could move vertically with compression. A quadrangle was selected as the mesh shape.

The behavior of the granules was represented as a DPC model. The DPC model and the parameters have been described in detail in the literature. ${ }^{14)}$ DPC model consists of some parameters to correspond with each powder behavior. In the many literatures, approximately five DPC model parameters were measured. ${ }^{3-5)}$ In this study, six kinds of parameters of the DPC model implemented in a commercial package,

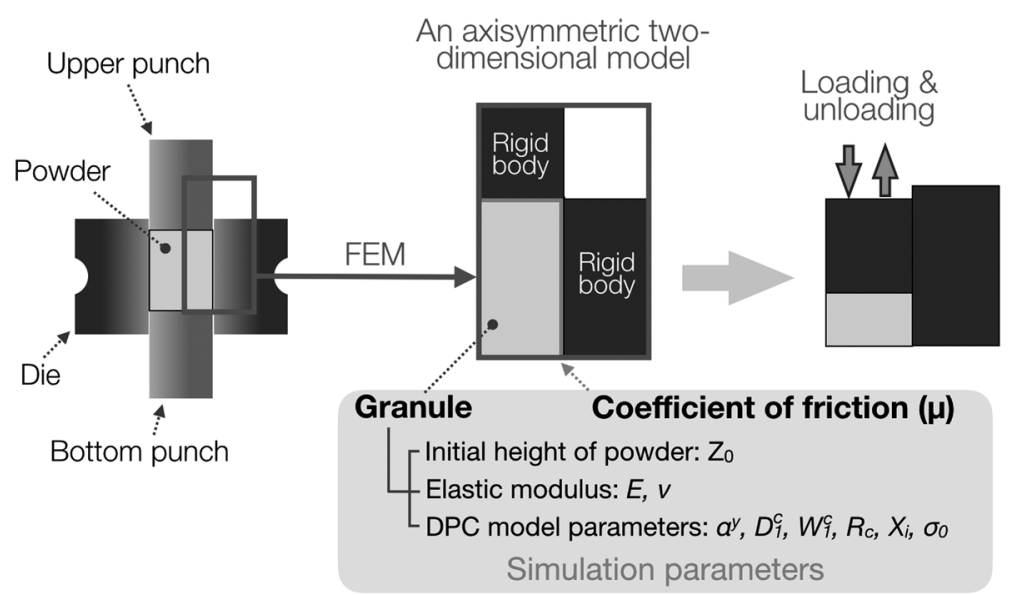

Fig. 1. A Typical Finite Element Model for Modeling the Compaction of Flat-Faced Tablets

The powders were modeled using the DPC model. Punch and die were considered as rigid bodies. An axisymmetric two-dimensional model (right half) was used. 
ANSYS, such as the parameter related to internal friction angle $\left(\alpha^{y}\right)$, the cohesion yield parameter $\left(\sigma_{0}\right)$, a cap shape parameter $\left(R_{c}\right)$, the initial value which the cap takes effect in the plasticity model $\left(X_{i}\right)$, the limiting value of volumetric plastic strain $\left(W_{1}^{c}\right)$ and the parameters that determine the shape of the pressure-volumetric strain curves $\left(D_{1}^{c}\right)$ were measured. The $\alpha^{y}, \sigma_{0}, R_{c}$ and $X_{i}$ are related to yield surface. The $W_{1}^{c}$ and $D_{1}^{c}$ are related to hardening mechanism and define the typical cap hardening law which has the exponential form proposed by Fossum and Fredrich. ${ }^{15)}$ Although there are the other parameters in ANSYS, they were considered as constants and determined based on previous study. ${ }^{14)}$ The DPC model parameters as a function of the density are important for accuracy and robustness in the FEM simulation. ${ }^{3)}$ However, the DPC model implemented in the commercially available software ANSYS could not manage the DPC model parameter as a function of the density. Thus, these values were necessarily fixed as constant.

Measurement of the Initial Height of Powders and Elastic Modulus The $Z_{0}$ was measured based on the volume of the gap space between upper and lower punches at $0.1 \mathrm{kN}$ based on the work of $\mathrm{Wu}$ et $a l^{4)}$ Two elastic parameters, namely Young's modulus $(E)$ and a Poisson ratio $(v)$, can be determined from the unloading curve of die compaction experiments. ${ }^{8}$ For this study of the direct shear test, using uniaxial compaction in a cylindrical die in the $z$-direction, the following assumptions were made:

$$
\begin{gathered}
\sigma_{1}=\sigma_{z} \\
\sigma_{2}=\sigma_{3}=\sigma_{\tau}
\end{gathered}
$$

where $\sigma_{1}, \sigma_{2}$ and $\sigma_{3}$ are the principal stress. The $\sigma_{z}$ and $\sigma_{\tau}$ values represent the vertical and radial stresses, respectively. We therefore have:

$$
\begin{gathered}
p=\frac{1}{3}\left(\sigma_{z}+2 \sigma_{z}\right) \\
\sqrt{J_{2}^{\prime}}=\frac{1}{\sqrt{3}}\left|\sigma_{z}-\sigma_{\tau}\right|
\end{gathered}
$$

where $p$ and $\sqrt{J_{2}^{\prime}}$ are the equivalent hydrostatic pressure stress and square root of the second invariant of the deviatoric stress, respectively. Elastic modulus was calculated based on the variation of axial stress with volume strain $\left(\varepsilon_{z}\right)$ and the stress path in the $p-\sqrt{J_{2}^{\prime}}$ plane for the compaction of granules. The $\varepsilon_{z}$ value was defined as a ratio of the powder height during compaction $(Z)$ to $Z_{0}: \varepsilon_{z}=\left|\ln \left(Z / Z_{0}\right)\right|{ }^{8)}$ The bulk modulus $K$ and shear modulus $G$ were defined as:

$$
\begin{aligned}
K+\frac{4}{3} G & =\frac{\left(\sigma_{z}^{m}-\sigma_{z}^{h}\right)}{\left(\varepsilon_{z}^{m}-\varepsilon_{z}^{h}\right)} \\
\frac{2 G}{\sqrt{3 K}} & =\frac{\sqrt{J_{2}^{\prime m}}}{p^{m}-p^{h}}
\end{aligned}
$$

where the superscripts $m$ and $h$ denote the values at points when the compression pressure indicates the maximum value

\begin{tabular}{|c|c|c|c|c|c|c|}
\hline \multicolumn{5}{|c|}{ Design variables } & \multicolumn{2}{|c|}{ Response variables } \\
\hline$R_{c}$ & $X_{i}$ & $\sigma_{0}$ & $\alpha^{y}$ & $\mu$ & DWF $(\mathrm{kN})$ & PTR (\%) \\
\hline 0.6 & -1 & 0.00091 & 0.229 & 0.13 & 3.094 & 93.47 \\
\hline 0.6 & -1 & 0.00091 & 0.265 & 0.16 & 2.770 & 92.82 \\
\hline 0.6 & -1 & 0.00910 & 0.229 & 0.16 & 3.092 & 91.99 \\
\hline 0.6 & -1 & 0.00910 & 0.265 & 0.13 & 2.772 & 94.15 \\
\hline 0.6 & -10 & 0.00091 & 0.229 & 0.16 & 3.150 & 91.84 \\
\hline 0.6 & -10 & 0.00091 & 0.265 & 0.13 & 2.826 & 94.04 \\
\hline 0.6 & -10 & 0.00910 & 0.229 & 0.13 & 3.154 & 93.35 \\
\hline 0.6 & -10 & 0.00910 & 0.265 & 0.16 & 2.823 & 92.69 \\
\hline 0.7 & -1 & 0.00091 & 0.229 & 0.16 & 3.169 & 91.79 \\
\hline 0.7 & -1 & 0.00091 & 0.265 & 0.13 & 2.849 & 93.99 \\
\hline 0.7 & -1 & 0.00910 & 0.229 & 0.13 & 3.171 & 93.31 \\
\hline 0.7 & -1 & 0.00910 & 0.265 & 0.16 & 2.847 & 92.63 \\
\hline 0.7 & -10 & 0.00091 & 0.229 & 0.13 & 3.236 & 93.17 \\
\hline 0.7 & -10 & 0.00091 & 0.265 & 0.16 & 2.903 & 92.48 \\
\hline 0.7 & -10 & 0.00910 & 0.229 & 0.16 & 3.231 & 91.63 \\
\hline 0.7 & -10 & 0.00910 & 0.265 & 0.13 & 2.907 & 93.87 \\
\hline
\end{tabular}
and $\sqrt{J_{2}^{\prime}}$ is 0 , respectively. Knowing the $K$ and $G$ values, the $E$ and $v$ values can be determined by:

$$
E=\frac{9 G K}{3 K+G}
$$

Table 1. Experimental Design for Screening Simulation Parameters

The values of DWF and PTR were calculated by FEM.

$$
v=\frac{3 K-2 G}{2(3 K+G)}
$$

Evaluation of an Influence of Simulation Parameters on Results The DoE was used to evaluate the relative contributions to the simulation parameters on the die wall force (DWF) and the pressure transmissibility rate of punches (PTR). Elastic modulus and the initial height of the powder bed were not taken into account because these parameters were obtained on the basis of experiments. The $W_{1}^{c}$ and $D_{1}^{c}$ values were not considered in the screening study because we have reported that these parameters are strongly affected by the volume strain and they could be estimated based on the variation of volume strain with compression. ${ }^{14)}$

Four DPC model parameters $\left(R_{c}, X_{i}, \sigma_{0}\right.$, and $\left.\alpha^{y}\right)$, and the $\mu$ value were selected as the causal factors influencing the DWF and the PTR. PTR was calculated by the following formula:

$$
\operatorname{PTR}(\%)=\frac{P_{L}}{P_{U}} \times 100
$$

where $P_{U}$ is the maximum pressure of the upper punch and $P_{L}$ is the maximum pressure of the lower punch in the tableting process.

Five parameters assigned to the L16 fractional factorial experimental design and 16 kinds of simulation conditions were constructed (Table 1). The compaction behavior of the powders during compaction was simulated using FEM according to DoE. To evaluate the significance of causal factors, the data obtained by FEM were analyzed by ANOVA and effect size was estimated. Two-way and three-way interaction effects were pooled. In this study, the omega-squared $\left(\omega^{2}\right)$ value was selected as the effect size ${ }^{16,17)}$ :

$$
\omega^{2}=\frac{\left(S S_{F}-\mathrm{d} f_{F} \times M S_{E}\right)}{S S_{T}+M S_{E}}
$$

where $S S_{F}$ and $S S_{T}$ are the sum of the square of each factor and total, respectively, $\mathrm{d} f_{F}$ is degrees of freedom of each factor, and $M S_{E}$ is the mean square of error. 
Estimation of the DPC Model Parameters and Coefficient of Friction The $\alpha^{y}$ and the $\mu$ value were fitted based on experimental results of compaction behavior. The two parameters assigned to $3^{2}$ full-factorial designs, and nine kinds of simulation conditions were constructed (Supplementary Table 1). The range of these parameters was determined using a preliminary trial. The tableting process was simulated using the FEM and estimated DWF and PTR values at maximum compaction $(6 \mathrm{kN})$. The data observed were modeled using the response surface method incorporating thin-plate spline interpolation (RSM-S) ${ }^{18)}$ The simulation parameters correlated with experiments were estimated based on the standardized Euclidian distance between the simulation and experimental values, and penalty functions. ${ }^{19,20)}$

In this study, the $W_{1}^{c}$ and $D_{1}^{c}$ were estimated in a similar way. The two parameters assigned $3^{2}$ full-factorial design and $\varepsilon_{z}$ values of each DPC model were estimated using FEM (Supplementary Tables 2,3) because the $W_{1}^{c}$ and $D_{1}^{c}$ values are related to volume change. ${ }^{21)}$ The range of these parameters was determined using a preliminary test. The $\varepsilon_{z}$ value was modeled as a function of two parameters based on RSM-S. The simultaneous optimum parameters were estimated on the basis of good agreement with $\varepsilon_{z}$ values in experiments at 1,2 , $3,4,5$, and $6 \mathrm{kN}$.

$R_{c}=0.6, X_{i}=-10$ and $\sigma_{0}=0.91$ were set as arbitrary values that did not cause divergence problems in the FEM because these parameters have a little influence on numerical results such as the PTR value, the DWF value and the volume change, while the other DPC model parameters have a limited impact on results (details are presented in Results and Discussion).

Computer Programs The FEM analysis of the tableting process was performed using ANSYS software, version 16.1 (ANSYS Inc., Canonsburg, PA, U.S.A.), in which the DPC model was implemented. The DoE, $t$-test and the ANOVA were performed using JMP Pro, version 11 (SAS Institute, Cary, NC, U.S.A.). The RSM-S was performed using dataNESIA, version 3.2 (Azbil Corporation, Tokyo, Japan).

\section{Results and Discussion}

Influence of Granule Size on Volume Strain, Thickness, DWF, PTR and Elastic Modulus The mechanical properties of small and large granules are summarized in Table 2. The $\varepsilon_{z}$, DWF, and PTR values during tableting were measured, and then compared between small and large granules. The average values for $\varepsilon_{z}, Z_{0}$, thickness of the tablet, PTR, and $E$ were smaller for small granules than for large granules.
By contrast, DWF and $v$ were higher for small granules than for large granules. Although these differences were relatively small, the differences in $\varepsilon_{z}, Z_{0}$, thickness of the tablet, PTR, and $v$ were significant.

Elastic recovery (so-called spring-back) of small granules was weaker than that of large granules. No significant differences between the different tablet thicknesses were observed at the maximum compression pressure, although the thickness of the tablets comprising small granules was smaller than that of tablets comprising large granules after ejection. The above result suggests that tablets composed of large granules are more porous than those composed of small granules. In other words, the inner structure of tablets composed of small or large granules is different. Moreover, the granule structure significantly affects the compaction procedure, resulting in different residual stress in the tablet.

The mean particle size is positively correlated to the PTR value, whereas DWF value is negatively correlated. Results observed in this study were consistent with previous findings reported by Otsuka et al. ${ }^{22)}$ The variation of PTR values might be related to the contact area between granules and the die/punch; that is, small granules have a higher contact area because of the higher specific surface area. Increasing the contact area between powders and the die/punch may result in lower PTR values. Otherwise, sufficient amounts of $\mathrm{Mg}-\mathrm{St}$ may not be composed of small granules. The specific surface area of small granules is greater than that of large granules so that much $\mathrm{Mg}-\mathrm{St}$ is needed compared with large granules. Therefore, the PTR of small granules is less than that of large granules.

Influence of Simulation Parameters on DWF and PTR The influence of five parameters $\left(R_{c}, X_{i}, \sigma_{0}, \alpha^{y}\right.$, and $\left.\mu\right)$ on the shear stress was evaluated. We estimated the value of $\omega^{2}$ in order to evaluate contributions of simulation parameters on simulation results. The $\omega^{2}$ value of simulation parameters is shown in Fig. 2. In DWF, the $\omega^{2}$ value of $\alpha^{y}$ indicated a high value such as 0.92 . The other parameters were relatively small, although a large effect gave the $\omega^{2}$ value above 0.14 in general. ${ }^{17)}$ These results suggested that the $\alpha^{y}$ value was a key parameter determining the DWF value. This result suggests that the DWF is linked with the internal friction between granules because the value of $\alpha^{y}$ is related to the internal friction. In PTR, the $\omega^{2}$ value in $\mu$ and $\alpha^{y}$ values were 0.76 and 0.22 , respectively, while these values in the other parameters were relatively small. These findings clearly indicate that the $\mu$ and $\alpha^{y}$ values strongly contribute to PTR. In particular,

Table 2. Comparison of Compaction Properties of Small Granules with Large Ones

\begin{tabular}{lccc}
\hline \hline & Small granules & Large granules & $p$ \\
\hline$\varepsilon_{z}$ at $160 \mathrm{MPa}$ & $0.371 \pm 0.003$ & $0.386 \pm 0.005$ & $0.008^{* *}$ \\
$Z_{0}$ & $4.078 \pm 0.03$ & $4.159 \pm 0.010$ & $0.007^{* *}$ \\
Thickness of tablet (mm) & $2.950 \pm 0.003$ & $2.969 \pm 0.002$ & $0.001^{* *}$ \\
DWF (kN) & $2.967 \pm 0.027$ & $2.918 \pm 0.033$ & 0.113 \\
PTR (\%) & $92.677 \pm 0.043$ & $93.375 \pm 0.113$ & $<0.001^{* *}$ \\
$E(\mathrm{GPa})$ & $2.717 \pm 0.118$ & $2.819 \pm 0.100$ & 0.317 \\
$v$ & $0.195 \pm 0.002$ & $0.188 \pm 0.002$ & $0.013^{*}$ \\
Minimum shear stress (MPa) & -5.294 & -4.671 & - \\
Maximum shear stress (MPa) & 0.060 & 0.155 & - \\
\hline
\end{tabular}

Each data point represents the mean \pm S.D. of three determinations. ** Indicates $p<0.01$, and *indicates $p<0.05$. As for minimum and maximum shear stress, a hypothesis test was not performed because these values were estimated by FEM. 


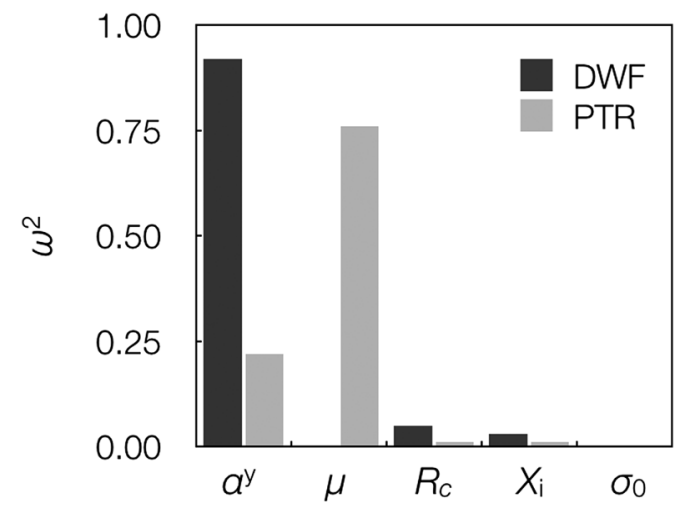

Fig. 2. The $\omega^{2}$ Values for Simulation Parameters Estimated in the Screening Study

$\omega^{2}$ indicates the impact of the simulation parameters on the DWF or PTR.

this result indicates that the PTR value is related to friction between granule and die/punch, and internal friction between granules. Although the detailed mechanism is unclear, $\alpha^{y}$ might affect volume strain of tablet toward die in the FEM simulation. Increasing volume strain toward die interferes transmissibility of the pressure from upper to bottom punch. Therefore, $\alpha^{y}$ has an influence on DWF and PTR. On the other hand, $\mu$ had no effect on volume strain toward die because $\mu$ is the boundary condition in the FEM simulation. As a result, we estimated $\alpha^{y}$ and $\mu$ values based on the experimental values of DWF and PTR.

Estimation of Simulation Parameters Using the Response Surface and Optimization Technique In general, the $\mu$ was calculated from the Janssen-Walker equation and considered as a constant in FEM simulation. ${ }^{2,3)}$ The value of $\mu$ converged during tableting was used for simulation. However, punch pressures of simulation and experiment have not been compared. ${ }^{2,3)}$ In this study, when compression pressure varied from 15 to $120 \mathrm{MPa}$, the $\mu$ values varied from 0.117 to 0.194 for small granules, and from 0.100 to 0.166 for large granules, respectively (Supplementary Fig. 1). When the $\mu$ values of small and large granules were set at 0.117 and 0.100 , respectively, the results from PTR simulation were not consistent with the experimental results. Therefore, to estimate the optimum values of $\mu$ within the range given by the Janssen-Walker equation, an optimization technique was used to construct a quantitative correlation model of simulation parameters and simulation results in this study.

The $\alpha^{y}$ and $\mu$ values were assigned to DoE, and the DWF and PTR values were calculated using the FEM (Supplementary Table 1). Figure 3 shows response surfaces for DWF and PTR as a function of $\alpha^{y}$ and $\mu$ values estimated by using RSM-S. The DWF value increased with increasing $\alpha^{y}$ values, but it was not affected by $\mu$ (Fig. 3a), suggesting that the DWF value increased with internal friction between granules. These results also indicate that DWF is driven only by $\alpha^{y}$ values, and were consistent with the screening study. By contrast, the PTR value was affected not only by the $\alpha^{y}$ value, but also by $\mu$. The PTR value increased with increasing $\alpha^{y}$ values and decreasing $\mu$ (Fig. 3b). In other words, the PTR value increased with increasing internal friction between granules and decreasing friction between granule and die/punch.

The $W_{1}^{c}$ and $D_{1}^{c}$ were assigned to DoE, and nine kinds of DPC models were constructed. The variation of $\varepsilon_{z}$ values with compression pressure was estimated by FEM (Supplementary Tables 2, 3). Using RSM-S, the data obtained were modeled as a function of $W_{1}^{c}$ and $D_{1}^{c}$ values. The larger $W_{1}^{c}$ and $D_{1}^{c}$ values led to increasing $\varepsilon_{z}$ values at $1 \mathrm{kN}$ (Fig. $3 \mathrm{c}$ ). By contrast, the $D_{1}^{c}$ value had no influence on the $\varepsilon_{z}$ value at $6 \mathrm{kN}$, whereas the $\varepsilon_{z}$ value increased with increasing $W_{1}^{c}$ (Fig. 3d). The $W_{1}^{c}$ value is the dominating factor that determines the maximum $\varepsilon_{z}$ value, because the $W_{1}^{c}$ is defined as the maximum plastic volumetric strain. ${ }^{24)}$ On the other hand, the $D_{1}^{c}$ value is parameters that determine the shape of the pressure-volumetric strain curves. ${ }^{15)}$ Therefore, it is likely that the $W_{1}^{c}$ value contributes to the $\varepsilon_{z}$ value in any compression force, although the $D_{1}^{c}$ value affects the $\varepsilon_{z}$ value only at low compression force.

We calculated the optimum values of $\mu, \alpha^{y}, W_{1}^{c}$, and $D_{1}^{c}$ based on optimization technique. Results are summarized in Table 3. The $\mu$ value of small granules was higher than that of large granules. The $\mu$ values suggested that higher friction between powder and die/punch was generated in the case of small granules, and this was reasonable because the PTR value of small granules was smaller than that of large granules. In general, the smaller $\alpha^{y}$ values indicated that tablets were more easily broken when the $\sigma_{0}$ value was the same. In this study, the $\alpha^{y}$ value of small and large granules were 0.2444 or 0.2474 , respectively. Consequently, almost no difference in $\alpha^{y}$ was observed between small and large granules. The $W_{1}^{c}$ values of small granules were also lower than those of large granules. As for the difference of $W_{1}^{c}$ values between granules, large granules showed a greater plastic strain compared with small granules, reflecting results observed in the $\varepsilon_{z}$ values. The same value was observed in regard to the $D_{1}^{c}$ value.

Comparison of Experimental and Simulation Results It is important to compare the simulation result to the experimental result to validate the model used for the simulation. ${ }^{7}$ We evaluated FEM results in the experiment to determine the adequacy of FEM simulation.

The compression pressure was shown as a function of $\varepsilon_{z}$ during the tableting of small and large granules (Fig. 4). The loading and unloading curves of the FEM simulation were highly consistent with experiments but were a little different at low compression pressure during loading. The $\varepsilon_{z}$ values from the FEM and from experiment for small granules at $6 \mathrm{kN}$ were 0.362 and $0.371 \pm 0.003$, respectively. In the case of large granules, the $\varepsilon_{z}$ values from the FEM and from experiment at $6 \mathrm{kN}$ were 0.385 and $0.386 \pm 0.005$, respectively. These results indicate that FEM simulation of volume change provides excellent reliability and the optimum $W_{1}^{c}$ and $D_{1}^{c}$ values were substantially appropriate.

We also compared the experimental and simulation values relating to the pressure during the compaction. DWF values of small granules in the FEM simulation and the experiment at $6 \mathrm{kN}$ were 2.976 and $2.967 \pm 0.027$, respectively. These values in the case of large granules were 2.918 and 2.918 \pm 0.033 , respectively. By contrast, the value of PTR from the FEM and experiment using small granules at $6 \mathrm{kN}$ were 92.674 and $92.677 \pm 0.043$, respectively. The value of PTR from the FEM and experiment using large granules at $6 \mathrm{kN}$ were 93.365 and 93.375 \pm 0.113 , respectively. The FEM simulation sufficiently reflected the experimental results and revealed the difference between the granules.

Residual Shear Stress Distribution of Each Tablet Fig- 
(a)

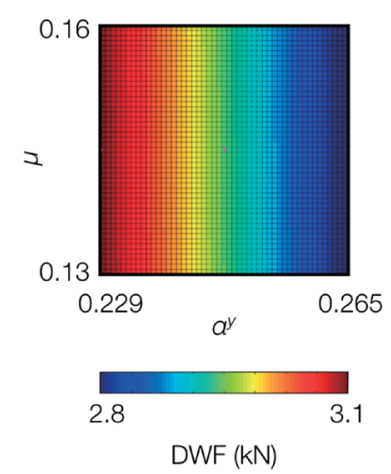

(b)

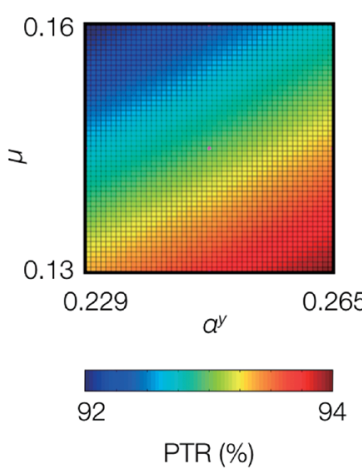

(c)

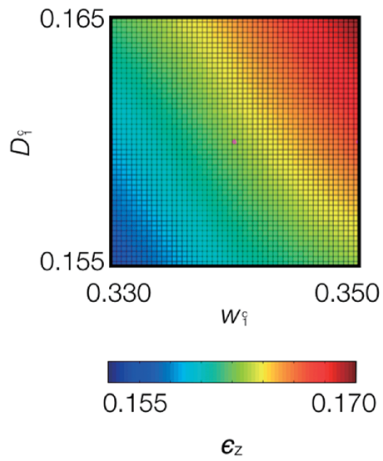

(d)

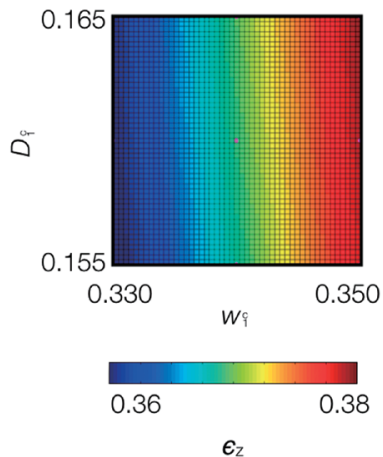

Fig. 3. Response Surfaces for (a) DWF, (b) PTR, (c) $\varepsilon_{z}$ at $1 \mathrm{kN}$, and (d) $\varepsilon_{z}$ at $6 \mathrm{kN}$ Estimated by RSM-S

DWF and PTR values were modeled as a function of $\alpha^{y}$ and $\mu$. Volume strain was modeled as a function of $W_{1}^{c}$ and $D_{1}^{c}$.

Table 3. Simulation Parameters of Small and Large Granules Estimated Based on Compaction Behavior and Optimization Technique

\begin{tabular}{lccc}
\hline \hline & \multicolumn{2}{c}{ Simulation parameters } & \multirow{2}{*}{$\omega^{2}$} \\
\cline { 2 - 3 } & Small granules & Large granules & \\
\hline$Z_{0}(\mathrm{~mm})$ & 4.0780 & 4.1650 & 0.00 \\
$\mu$ & 0.1524 & 0.1402 & 0.97 \\
$E(\mathrm{GPa})$ & 2.7170 & 2.8190 & 0.01 \\
$v$ & 0.1952 & 0.1881 & 0.00 \\
$\alpha^{y}$ & 0.2444 & 0.2474 & 0.02 \\
$W_{1}^{c}$ & 0.3350 & 0.3560 & 0.00 \\
$D_{1}^{c}$ & 0.0165 & 0.0165 & - \\
\hline
\end{tabular}

The omega-squared indicates an influence of simulation parameters on the minimum residual shear stress of the tablet. Results were observed using an L16 fractional factorial design and an ANOVA to the simulation data of the FEM.

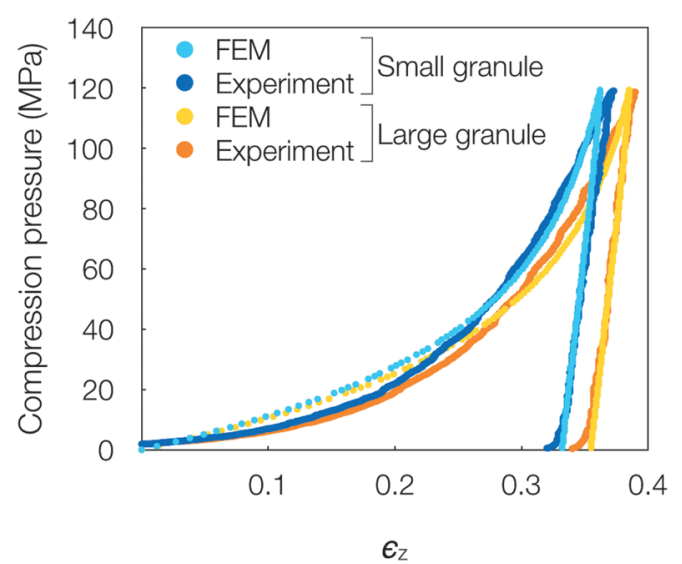

Fig. 4. Comparison between Experimental Results and Simulation of Small and Large Granules

The variation of axial strain with compression pressure.

(b)

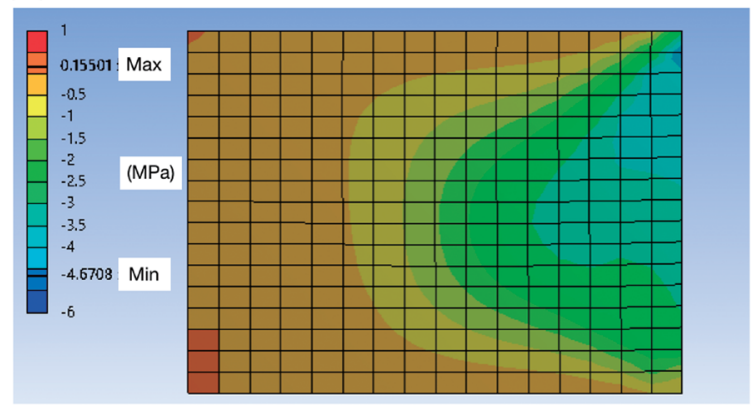

Fig. 5. Two-Dimensional Maps for the Residual Shear Stress Distribution of Tablets Composed of (a) Small and (b) Large Granules Estimated Using the FEM

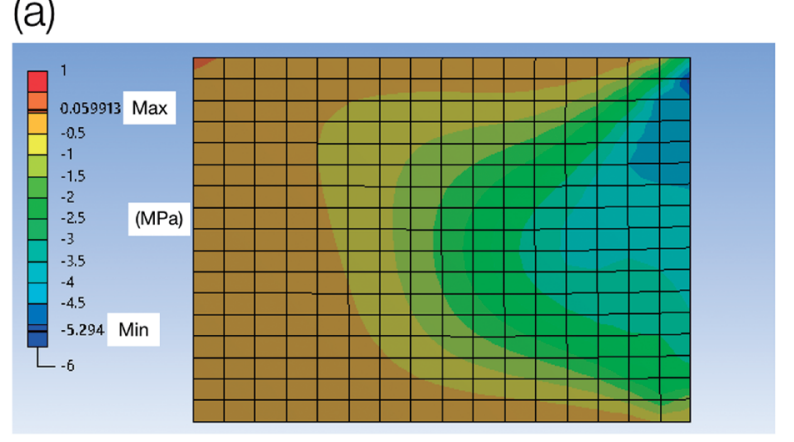

The result represents the right-half of the tablet.

ure 5 depicts two-dimensional maps for the residual shear stress distribution of tablets composed of small and large granule estimated by the FEM simulation. The trend for the residual stress distribution was similar in the granules. An intensive shear region was recognized from the top edge to the mid center of tablet. In this shear region, the shear stress changed from positive to negative, indicating a change in the direction of the shear stress. These phenomena are more likely to be induced by spring-back during unloading. After maximum compres- sion pressure, the distance between the two punches increased, resulting in an increase in the volume of the powder bed. FEM analysis indicates that the powder near the top will generally relax more than that at the bottom because of the friction generated between the die and the powder.

The minimum and maximum residual shear stresses of small and large granule are summarized in Table 2. Compared with values for residual shear stress, the minimum residual shear stress was slightly lower for small granules than for 
large granules. Moreover, the difference between minimum and maximum shear stresses of small granules was always greater than that of large granules during tableting. This indicates that small granules induce greater stress in the tablet during and after tableting. Generally, a granulation process is performed to prevent segregation of the constituents of the powder mixture, and to improve flow properties and compaction characteristics in pharmaceutical products. ${ }^{25)}$ Decreasing the shear stress of tablets might be one of the factors those improve the compaction characteristics.

Contribution of Simulation Parameters on Residual Shear Stress Distribution To evaluate the causal relationships between granule size, simulation parameters, and shear stress, further simulation was conducted. Six simulation parameters: $Z_{0}, \mu, E, v, \alpha^{y}$, and $W_{1}^{c}$ were assigned according to DoE (an L16 fractional factorial design) and an ANOVA was applied to DoE results. The $D_{1}^{c}$ value was excluded in this study because this parameter remained invariant. The range of the parameters was determined based on the value of small and large granules. The $\omega^{2}$ value was applied to evaluating the relative effect of deviation of parameters on the simulation results.

The $\omega^{2}$ value of each factor is summarized in Table 3. The $\omega^{2}$ values of $\mu, \alpha^{y}$, and $E$ were $0.97,0.02$, and 0.01 , respectively, and the others were 0.00 . This result suggested that the effect of $\mu$ is overwhelmingly greater than that of the other parameters. Therefore, our results suggested that $\mu$ was the critical parameter affecting the residual shear stress when the small and large granules were compressed. Sinka et al. ${ }^{2)}$ investigated the difference between lubricated and clean dies. They reported that the $\mu$ value affected the relative density distribution of tablets. Moreover, Garner et al. ${ }^{26)}$ conducted a sensitivity analysis of shear failure parameters in a finite element compaction model. They reported that these parameters had little to no effect on die compaction model results. Therefore, our results are consistent with the findings described above.

\section{Conclusion}

The aim of this study was to evaluate relationships between the granule size, simulation parameters, and the residual shear stress of tablets. Influence of granule size on the compaction behavior was investigated using a single tableting machine equipped with an instrumented die. DoE and RSM-S revealed the quantitative relationships between simulation parameters and tableting characteristics such as DWF, PTR values, and the volume strain. The compaction behavior simulated by the FEM was in reasonable agreement with the experiments, although a slight difference was observed at unloading of the stress path. FEM analysis revealed tablets composed of small granules were more inhomogeneous than those composed of large granules. To understand the mechanism, the impact of simulation parameters on residual stress was investigated using DoE and ANOVA. The $\mu$ value was one of the most important factors governing residual shear stress. This study also demonstrated the importance of the combined use of FEM simulation and statistical analysis to gain a deeper understanding of tableting processes.

Acknowledgments This study was supported by JSPS KAKENHI Grant Numbers JP16K08192 and JP17K08192. We thank Dr. Hidemasa Nagai and Mr. Isao Myokan at Toyama
Prefectural Institute for Pharmaceutical Research for their technical assistance.

Conflict of Interest The authors declare that they have no financial or noncompeting interests concerning this manuscript. The Department of Pharmaceutical Technology, University of Toyama, is an endowed department, supported by an unrestricted grant from Nichi-Iko Pharmaceutical Co. (Toyama, Japan).

Supplementary Materials The online version of this article contains supplementary materials.

\section{References}

1) Leuenberger H., Puchkov M., Schneider B., Swiss J. Pharm. Ind., 35, 3-16 (2013).

2) Sinka I. C., Cunningham J. C., Zavaliangos A., J. Pharm. Sci., 93, 2040-2053 (2004).

3) Han L. H., Elliott J. A., Bentham A. C., Mills A., Amidon G. E., Hancock B. C., Int. J. Solids Struct., 45, 3088-3106 (2008).

4) Wu C. Y., Ruddy O. M., Bentham A. C., Hancock B. C., Best S. M., Elliott J. A., Powder Technol., 152, 107-117 (2005).

5) Furukawa R., Chen Y., Horiguchi A., Takagaki K., Nishi J., Konishi A., Shirakawa Y., Sugimoto M., Narisawa S., Int. J. Pharm., 493, 182-191 (2015).

6) Mazel V., Diarra H., Busignies V., Tchoreloff P., J. Pharm. Sci., 104, 4339-4344 (2015).

7) Diarra H., Mazel V., Boillon A., Rehault L., Busignies V., Bureau S., Tchoreloff P., Powder Technol., 224, 233-240 (2012).

8) Aydin Í., Briscoe B. J., Şanlitürk K. Y., Powder Technol., 89, 239254 (1996).

9) Hörmann T., Suzzi D., Adam S., Khinast J. G., J. Pharm. Innov., 7, 181-194 (2012).

10) Hayashi Y., Otoguro S., Miura T., Onuki Y., Obata Y., Takayama K., Chem. Pharm. Bull., 62, 1062-1072 (2014).

11) Ohno I., Hasegawa S., Yada S., Kusai A., Moribe K., Yamamoto K., Int. J. Pharm., 338, 79-86 (2007).

12) Eichie F. E., Kudehinbu A. O., Afr. J. Biotechnol., 21, 5913-5916 (2009).

13) Norioka T., Hayashi Y., Onuki Y., Andou H., Tsunashima D., Yamashita K., Takayama K., Chem. Pharm. Bull., 61, 39-49 (2013).

14) Hayashi Y., Miura T., Shimada T., Onuki Y., Obata Y., Takayama K., J. Pharm. Sci., 102, 3678-3686 (2013).

15) Fossum A., Fredrich J., Pacific Rocks 2000 Rock Around Rim. Proc. Fourth North Am. Rock Mech. Symp., 1169-1176 (2000).

16) Olejnik S., Algina J., Psychol. Methods, 8, 434-447 (2003).

17) Wang Y., Snee R. D., Keyvan G., Muzzio F. J., Drug Dev. Ind. Pharm., 9045, 1-12 (2015).

18) Arai H., Suzuki T., Kaseda C., Ohyama K., Takayama K., Chem. Pharm. Bull., 55, 586-593 (2007).

19) Takayama K., Nagai T., Int. J. Pharm., 74, 115-126 (1991).

20) Khuri A. I., Conlon M., Technometrics, 23, 363-375 (1981)

21) Hayashi Y., Miura T., Shimada T., Onuki Y., Obata Y., Takayama K., J. Pharm. Sci., 102, 3678-3686 (2013).

22) Otsuka T., Iwao Y., Miyagishima A., Itai S., Int. J. Pharm., 409, 81-88 (2011).

23) Cunningham J. C., Sinka I. C., Zavaliangos A., J. Pharm. Sci., 93, 2022-2039 (2004).

24) Schwer L. E., Murray Y. D., Int. J. Numer. Anal. Methods Geomech., 18, 657-688 (1994).

25) Aulton M. E., Taylor K. M. G., "Aulton's Pharmaceutics," 4th ed., Elsevier, Amsterdam, 2013.

26) Garner S., Strong J., Zavaliangos A., Powder Technol., 283, 210 226 (2015). 